\title{
ON HUPPERT'S CONJECTURE FOR THE CONWAY AND FISCHER FAMILIES OF SPORADIC SIMPLE GROUPS
}

\author{
S. H. ALAVI, A. DANESHKHAH ${ }^{\otimes}$, H. P. TONG-VIET and T. P. WAKEFIELD \\ (Received 29 August 2011; accepted 18 May 2012; first published online 11 April 2013) \\ Communicated by E. A. O’Brien
}

\begin{abstract}
Let $G$ denote a finite group and $\operatorname{cd}(G)$ the set of irreducible character degrees of $G$. Huppert conjectured that if $H$ is a finite nonabelian simple group such that $\operatorname{cd}(G)=\operatorname{cd}(H)$, then $G \cong H \times A$, where $A$ is an abelian group. He verified the conjecture for many of the sporadic simple groups and we complete its verification for the remainder.
\end{abstract}

2010 Mathematics subject classification: primary 20C15; secondary 20D05.

Keywords and phrases: character degrees, sporadic simple groups, Huppert's conjecture.

\section{Introduction}

Let $G$ be a finite group, $\operatorname{Irr}(G)$ the set of irreducible characters of $G$, and denote the set of character degrees of $G$ by $\operatorname{cd}(G)=\{\chi(1): \chi \in \operatorname{Irr}(G)\}$. This set can be used to gain information about the structure of $G$. For example, it can be used to determine if $G$ is abelian. Unfortunately, it does not completely determine the structure of $G$. It is possible for nonisomorphic groups to have the same set of character degrees. For example, the nonisomorphic groups $D_{8}$ and $Q_{8}$ not only have the same set of character degrees, but also share the same character table. The character degree set cannot be used to distinguish between solvable and nilpotent groups. For example, if $G$ is either $Q_{8}$ or $S_{3}$, then $\operatorname{cd}(G)=\{1,2\}$. However, in the late 1990s, Huppert [6] posed a conjecture which, if true, would sharpen the connection between the character degree set of a nonabelian simple group and the structure of the group.

Conjecture 1.1 (Huppert). Let $G$ be a finite group and $H$ a finite nonabelian simple group such that the sets of character degrees of $G$ and $H$ are the same. Then $G \cong H \times A$, where $A$ is an abelian group.

The third author is financially supported by NRF, NWU (Mafikeng) and UKZN.

(C) 2013 Australian Mathematical Publishing Association Inc. 1446-7887/2013 \$16.00 
The conjecture asserts that the nonabelian simple groups are essentially characterized by the set of their character degrees. It does not extend to solvable groups. Unfortunately, it is not possible to establish the conjecture for all nonabelian simple groups with a single argument. In addition to verifying this conjecture for many of the simple groups of Lie type, Huppert also verified it for many of the sporadic simple groups. The sporadic simple groups not considered by him are Conway groups, Fischer groups, the Monster, and the Baby Monster. The conjecture for the Baby Monster, the Monster and the Fischer group $\mathrm{Fi}_{23}$ was established in [1, 8]. Huppert's proofs rely upon establishing five steps to achieve the result. Steps 2 and 3 are the most delicate and require the most specific and technical arguments.

(1) Show that $G^{\prime}=G^{\prime \prime}$. Hence if $G^{\prime} / M$ is a chief factor of $G$, then $G^{\prime} / M \cong S^{k}$, where $S$ is a nonabelian simple group.

(2) Identify $H$ as a chief factor $G^{\prime} / M$ of $G$.

(3) Show that if $\theta \in \operatorname{Irr}(M)$ and $\theta(1)=1$, then $\theta^{g}=\theta$ for all $g \in G^{\prime}$, (that is, $\theta$ is stable under $G^{\prime}$ ), which implies that $\left[M, G^{\prime}\right]=M^{\prime}$.

(4) Show that $M=1$.

(5) Show that $G=G^{\prime} \times C_{G}\left(G^{\prime}\right)$. As $G / G^{\prime} \cong C_{G}\left(G^{\prime}\right)$ is abelian and $G^{\prime} \cong H$, Huppert's conjecture is verified.

We utilize these steps to verify the conjecture for the remaining sporadic simple groups.

\section{Preliminaries}

In this section, we present some necessary results to establish the conjecture for the Conway and Fischer families of simple groups.

If $n$ is an integer, let $\pi(n)$ denote the set of all prime divisors of $n$. If $G$ is a group, we write $\pi(G)$ instead of $\pi(|G|)$ to denote the set of all prime divisors of the order of $G$. If $N \unlhd G$ and $\theta \in \operatorname{Irr}(N)$, then the inertia group of $\theta$ in $G$ is $I_{G}(\theta)=\left\{g \in G \mid \theta^{g}=\theta\right\}$. If the character $\chi=\sum_{i=1}^{k} e_{i} \chi_{i}$, where each $\chi_{i}$ is an irreducible character of $G$ and $e_{i}$ is a nonnegative integer, then those $\chi_{i}$ with $e_{i}>0$ are called the irreducible constituents of $\chi$. The set of all irreducible constituents of $\theta^{G}$ is denoted by $\operatorname{Irr}(G \mid \theta)$. Other notation is standard.

Lemma 2.1 [5, Theorems 19.5 and 21.3]. Suppose that $N \unlhd G$ and $\chi \in \operatorname{Irr}(G)$.

(a) If $\chi_{N}=\theta_{1}+\theta_{2}+\cdots+\theta_{k}$ with $\theta_{i} \in \operatorname{Irr}(N)$, then $k$ divides $|G / N|$. In particular, if $\chi(1)$ is prime to $|G / N|$, then $\chi_{N} \in \operatorname{Irr}(N)$.

(b) (Gallagher's theorem) If $\chi_{N} \in \operatorname{Irr}(N)$, then $\chi \psi \in \operatorname{Irr}(G)$ for all $\psi \in \operatorname{Irr}(G / N)$.

Lemma 2.2 [5, Theorems 19.6 and 21.2]. Suppose that $N \unlhd G$ and $\theta \in \operatorname{Irr}(N)$. Let $I=$ $I_{G}(\theta)$.

(a) If $\theta^{I}=\sum_{i=1}^{k} \varphi_{i}$ with $\varphi_{i} \in \operatorname{Irr}(I)$, then $\varphi_{i}^{G} \in \operatorname{Irr}(G)$. In particular, $\varphi_{i}(1)|G: I| \in$ $\operatorname{cd}(G)$.

(b) If $\theta$ extends to $\psi \in \operatorname{Irr}(I)$, then $(\psi \tau)^{G} \in \operatorname{Irr}(G)$ for all $\tau \in \operatorname{Irr}(I / N)$. In particular, $\theta(1) \tau(1)|G: I| \in \operatorname{cd}(G)$. 
(c) If $\rho \in \operatorname{Irr}(I)$ such that $\rho_{N}=e \theta$, then $\rho=\theta_{0} \tau_{0}$, where $\theta_{0}$ is a character of an irreducible projective representation of I of degree $\theta(1)$ and $\tau_{0}$ is a character of an irreducible projective representation of $I / N$ of degree $e$.

The following lemma [8, Lemma 3] will be used to verify step 1 .

Lemma 2.3. Let $G / N$ be a solvable factor group of $G$ minimal with respect to being nonabelian. Then two cases can occur.

(a) $G / N$ is an $r$-group for some prime $r$. Hence there exists $\psi \in \operatorname{Irr}(G / N)$ such that $\psi(1)=r^{b}>1$. If $\chi \in \operatorname{Irr}(G)$ and $r \nmid \chi(1)$, then $\chi \tau \in \operatorname{Irr}(G)$ for all $\tau \in \operatorname{Irr}(G / N)$.

(b) $G / N$ is a Frobenius group with an elementary abelian Frobenius kernel $F / N$. Then $f=|G: F| \in \operatorname{cd}(G)$ and $|F / N|=r^{a}$ for some prime $r$, and $a$ is the smallest integer such that $r^{a} \equiv 1 \bmod f$. If $\psi \in \operatorname{Irr}(F)$ then either $f \psi(1) \in \operatorname{cd}(G)$ or $r^{a}$ divides $\psi(1)^{2}$. In the latter case, $r$ divides $\psi(1)$.

(1) If no proper multiple of $f$ is in $\operatorname{cd}(G)$, then $\chi(1)$ divides $f$ for all $\chi \in \operatorname{Irr}(G)$ such that $r \nmid \chi(1)$, and if $\chi \in \operatorname{Irr}(G)$ such that $\chi(1) \nmid f$, then $r^{a} \mid \chi(1)^{2}$.

(2) If $\chi \in \operatorname{Irr}(G)$ such that no proper multiple of $\chi(1)$ is in $\operatorname{cd}(G)$, then either $f$ divides $\chi(1)$ or $r^{a}$ divides $\chi(1)^{2}$. Moreover, if $\chi(1)$ is divisible by no nontrivial proper character degree in $G$, then $f=\chi(1)$ or $r^{a} \mid \chi(1)^{2}$.

Let $\chi \in \operatorname{Irr}(G)$. We say that $\chi$ is isolated in $G$ if $\chi(1)$ is divisible by no proper nontrivial character degree of $G$ and no proper multiple of $\chi(1)$ is a character degree of $G$. In this situation, we also say that $\chi(1)$ is an isolated degree of $G$. We define a proper power degree of $G$ to be a character degree of $G$ of the form $f^{a}$ for integers $f, a>1$. Throughout this paper we use GAP [4] to determine the isolated degrees of the groups under discussion.

The next two lemmas will be used to verify steps 2 and 4 . The first lemma appears in [2, Theorems 2-4].

LEMma 2.4. If $S$ is a nonabelian simple group, then there exists a nontrivial irreducible character $\theta$ of $S$ that extends to $\operatorname{Aut}(S)$. Moreover, the following holds.

(a) If $S$ is an alternating group of degree at least seven, then $S$ has two characters of consecutive degrees $n(n-3) / 2$ and $(n-1)(n-2) / 2$ that both extend to $\operatorname{Aut}(S)$.

(b) If $S$ is a sporadic simple group or the Tits group, then $S$ has two nontrivial irreducible characters of coprime degrees which both extend to $\operatorname{Aut}(S)$.

(c) If $S$ is a simple group of Lie type, then the Steinberg character $S t_{S}$ of $S$ of degree $|S|_{p}$ extends to $\operatorname{Aut}(S)$.

Lemma 2.5 [2, Lemma 5]. Let $N$ be a minimal normal subgroup of $G$ so that $N \cong S^{k}$, where $S$ is a nonabelian simple group. If $\theta \in \operatorname{Irr}(S)$ extends to $\operatorname{Aut}(S)$, then $\theta^{k} \in \operatorname{Irr}(N)$ extends to $G$.

The following lemma will be used to verify step 4 . 
Lemma 2.6 [6, Lemma 6]. Suppose that $M \unlhd G^{\prime}=G^{\prime \prime}$ and for every $\lambda \in \operatorname{Irr}(M)$ with $\lambda(1)=1, \lambda^{g}=\lambda$ for all $g \in G^{\prime}$. Then $M^{\prime}=\left[M, G^{\prime}\right]$ and $\left|M / M^{\prime}\right|$ divides the order of the Schur multiplier of $G^{\prime} / M$.

\section{Huppert's conjecture for the sporadic simple group $\mathbf{F i}_{24}^{\prime}$}

3.1. The sporadic simple group $\mathbf{F i}_{\mathbf{2 4}}^{\prime}$. Using [3], we provide relevant properties of the sporadic simple group $H=\mathrm{Fi}_{24}^{\prime}$. Observe that $\pi(H)=\{2,3,5,7,11,13,17,23,29\}$.

Lemma 3.1. Let $H$ be the sporadic simple group $\mathrm{Fi}_{24}^{\prime}$.

(a) The following are isolated degrees of $\mathrm{H}$ :

$$
\begin{gathered}
3^{14} \cdot 7^{2} \cdot 23 \cdot 29, \quad 2^{14} \cdot 5^{2} \cdot 7 \cdot 11 \cdot 13 \cdot 17 \cdot 23, \\
2^{21} \cdot 5^{2} \cdot 7^{3} \cdot 11, \quad 3^{14} \cdot 17 \cdot 23 \cdot 29, \quad 3^{16} \cdot 11 \cdot 13 \cdot 29
\end{gathered}
$$

(b) Let $1 \neq \chi(1) \in \operatorname{cd}(H)$. Then $\operatorname{gcd}(11 \cdot 13 \cdot 23, \chi(1))>1$.

(c) The group $H$ has neither proper power degrees nor consecutive degrees.

(d) If $K$ is a maximal subgroup of $H$ such that $|H: K|$ divides some character degree $\chi(1)$ of $H$, then $K \cong \mathrm{Fi}_{23}$ and $\chi(1) /|H: K|$ divides one of the numbers in the set

$$
\begin{gathered}
\mathcal{A}=\left\{2^{4} \cdot 5^{2} \cdot 7 \cdot 17 \cdot 23,2 \cdot 3^{3} \cdot 7 \cdot 11 \cdot 13 \cdot 17,2^{2} \cdot 3 \cdot 11 \cdot 13 \cdot 17 \cdot 23,\right. \\
\left.2^{3} \cdot 3 \cdot 7 \cdot 11 \cdot 13 \cdot 23,2^{4} \cdot 3 \cdot 13 \cdot 17 \cdot 23,2 \cdot 7 \cdot 11 \cdot 17 \cdot 23\right\} .
\end{gathered}
$$

(e) The Schur multiplier $M\left(\mathrm{Fi}_{24}^{\prime}\right) \cong \mathbb{Z}_{3}$ and outer automorphism group $\operatorname{Out}\left(\mathrm{Fi}_{24}^{\prime}\right) \cong$ $\mathbb{Z}_{2}$.

3.2. Verifying Huppert's conjecture for $\mathbf{F i}_{24}^{\prime}$. We assume that $H \cong \mathrm{Fi}_{24}^{\prime}$ and $G$ is a group such that $\operatorname{cd}(G)=\operatorname{cd}(H)$. We will show that $G \cong H \times A$, where $A$ is an abelian group, which confirms Huppert's conjecture for $\mathrm{Fi}_{24}^{\prime}$. We follow the five steps of Huppert's method described in the introduction.

3.2.1. Verifying step 1. By way of contradiction, suppose that $G^{\prime} \neq G^{\prime \prime}$. Then there exists a normal subgroup $N \unlhd G$ where $N$ is maximal such that $G / N$ is solvable and nonabelian. By Lemma 2.3, $G / N$ is an $r$-group for some prime $r$ or $G / N$ is a Frobenius group.

Case 1. $G / N$ is an $r$-group. Then there exists $\psi \in \operatorname{Irr}(G / N)$ such that $\psi(1)=r^{b}>1$. By Lemma 3.1(c), $G$ has no nontrivial prime power degrees. Hence this case cannot happen.

Case 2. $G / N$ is a Frobenius group with Frobenius kernel $F / N,|F / N|=r^{a}, 1<f=\mid G$ : $F \mid \in \operatorname{cd}(G)$ and $r^{a} \equiv 1 \bmod f$. By Lemma 2.3(b), if $\chi \in \operatorname{Irr}(G)$ such that $\chi(1)$ is isolated, then either $f=\chi(1)$ or $r \mid \chi(1)$. We observe that there is no prime which divides all the isolated degrees listed in Lemma 3.1(a). Thus $f$ must be one of the isolated degrees in Lemma 3.1(a). By Lemma 2.3(b) again, if $\chi \in \operatorname{Irr}(G)$ with $r \nmid \chi(1)$, then $\chi(1) \mid f$. As $f$ is isolated we deduce that $r$ must divide every nontrivial degree $\chi(1)$ of $G$ such that $\chi(1) \neq f$. 
Assume first that $f=3^{14} \cdot 7^{2} \cdot 23 \cdot 29$. Then $r$ must divide all of the remaining isolated degrees in Lemma 3.1(a). As $\operatorname{gcd}\left(2^{21} \cdot 5^{2} \cdot 7^{3} \cdot 11,3^{14} \cdot 17 \cdot 23 \cdot 29\right)=1$, we find that $f \neq 3^{14} \cdot 7^{2} \cdot 23 \cdot 29$ and $r \in\{3,7,23,29\}$. For each value of $r$, there exist two distinct isolated degrees in Lemma 3.1(a) which are both prime to $r$, so $f$ must be equal to both by Lemma 2.3(b)(2), which is impossible. Thus $G^{\prime}=G^{\prime \prime}$.

3.2.2. Verifying step 2. Let $M \leq G^{\prime}$ be a normal subgroup of $G$ such that $G^{\prime} / M$ is a chief factor of $G$. As $G^{\prime}$ is perfect, $G^{\prime} / M$ is nonabelian. Hence $G^{\prime} / M \cong S^{k}$ for some nonabelian simple group $S$ and some integer $k \geq 1$.

Claim 1. $k=1$. By way of contradiction, assume that $k \geq 2$. By Lemma 2.4, $S$ possesses a nontrivial irreducible character $\theta$ extendible to $\operatorname{Aut}(S)$. By Lemma 2.5, $\theta^{k} \in \operatorname{Irr}\left(G^{\prime} / M\right)$ extends to $G / M$, hence $\theta(1)^{k} \in \operatorname{cd}(G)$, which contradicts Lemma 3.1(c). Hence $k=1$ and $G^{\prime} / M \cong S$.

Claim 2. $S$ is not an alternating group of degree at least seven. By way of contradiction, assume that $S=\mathrm{A}_{n}, n \geq 7$. By Lemma 2.4, $S$ has nontrivial irreducible characters $\theta_{1}, \theta_{2}$ with $\theta_{1}(1)=n(n-3) / 2, \theta_{2}(1)=\theta_{1}(1)+1=(n-1)(n-2) / 2$ and both $\theta_{i}$ extend to $\operatorname{Aut}(S)$. Thus $G$ possesses two consecutive nontrivial character degrees, contradicting Lemma 3.1(c).

Claim 3. $S$ is not a simple group of Lie type. If $S$ is a simple group of Lie type in characteristic $p$, and $S \neq{ }^{2} \mathrm{~F}_{4}(2)^{\prime}$, then the Steinberg character of $S$ of degree $|S|_{p}$ extends to $\operatorname{Aut}(S)$ so that $G$ possesses a nontrivial prime power degree, which contradicts Lemma 3.1(c).

Claim 4. $S \cong \mathrm{Fi}_{24}^{\prime}$. By Claims $1-3, S$ is a sporadic simple group or the Tits group. We will eliminate all other possibilities for $S$ and hence the claim will follow. By the Ito-Michler theorem (see [6, Lemma 1]) we deduce that every prime divisor of $S$ must divide some character degree of $S$ and, as every character degree of $S \cong G^{\prime} / M$ divides some character degree of $H$, every prime divisor of $S$ is also a prime divisor of $H$. Thus $\pi(S) \subseteq \pi(H)=\{2,3,5,7,11,13,17,23,29\}$. Hence we only need to consider the simple groups in Table 1 . For each group $S$ in Table 1, we exhibit a nontrivial irreducible character $\theta$ of $S$ such that $\theta$ extends to $\operatorname{Aut}(S)$ and $\operatorname{gcd}(11 \cdot 13 \cdot 23, \theta(1))=1$, which contradicts Lemma 3.1(b). This finishes the proof of step 2.

3.2.3. Verifying step 3. Let $\theta \in \operatorname{Irr}(M)$ with $\theta(1)=1$ and let $I=I_{G^{\prime}}(\theta)$. Assume that $I<G^{\prime}$ and $\theta^{I}=\sum_{i=1}^{s} e_{i} \phi_{i}$, where $\phi_{i} \in \operatorname{Irr}(I)$ for $i=1,2, \ldots, s$. Let $U / M$ be a maximal subgroup of $G^{\prime} / M$ containing $I / M$ and let $t=|U: I|$. Then $\phi_{i}(1)\left|G^{\prime}: I\right|$ is a character degree of $G^{\prime}$ by Lemma 2.2(a), so it divides some character degree of $G$. Thus $t \phi_{i}(1)\left|G^{\prime}: U\right|$ divides some character degree of $G$ and so the index $\left|G^{\prime}: U\right|$ must divide some character degree of $H$. By Lemma 3.1(d), $U / M \cong \mathrm{Fi}_{23}$ and, for each $i$, $t \phi_{i}(1)$ divides one of the numbers in $\mathcal{A}$. By inspecting the list of maximal subgroups of $\mathrm{Fi}_{23}$ in [3], the index of a maximal subgroup of $U / M$ divides no number in $\mathcal{A}$ so that $t=1$ and hence $I=U$. As the Schur multiplier of $I / M \cong \mathrm{Fi}_{23}$ is trivial and $\theta$ 
TABLE 1. Some character degrees of sporadic simple groups and Tits group.

\begin{tabular}{llrllr}
\hline Group & Character & Degree & Group & Character & Degree \\
\hline $\mathrm{M}_{11}$ & $\chi_{9}$ & $3^{2} \cdot 5$ & $\mathrm{~J}_{2}$ & $\chi_{6}$ & $2^{2} \cdot 3^{2}$ \\
$\mathrm{M}_{12}$ & $\chi_{6}$ & $3^{2} \cdot 5$ & $\mathrm{Co}_{3}$ & $\chi_{32}$ & $2^{7} \cdot 3^{6}$ \\
$\mathrm{M}_{22}$ & $\chi_{3}$ & $3^{2} \cdot 5$ & $\mathrm{Fi}_{22}$ & $\chi_{54}$ & $2^{5} \cdot 3^{5} \cdot 5^{2} \cdot 7$ \\
$\mathrm{M}_{23}$ & $\chi_{3}$ & $3^{2} \cdot 5$ & $\mathrm{HS}$ & $\chi_{24}$ & $2^{7} \cdot 5^{2}$ \\
$\mathrm{M}_{24}$ & $\chi_{3}$ & $3^{2} \cdot 5$ & $\mathrm{Co}_{1}$ & $\chi_{71}$ & $2^{18} \cdot 3^{6}$ \\
$\mathrm{McL}$ & $\chi_{12}$ & $2^{2} \cdot 3^{2} \cdot 5^{3}$ & ${ }^{2} \mathrm{~F}_{4}(2)^{\prime}$ & $\chi_{20}$ & $2^{6} \cdot 3^{3}$ \\
$\mathrm{He}$ & $\chi_{12}$ & $2^{7} \cdot 3 \cdot 5$ & $\mathrm{Suz}_{43}$ & $\chi_{43}$ & $2^{10} \cdot 3^{5}$ \\
$\mathrm{Co}_{2}$ & $\chi_{22}$ & $3^{6} \cdot 5^{3}$ & $\mathrm{Fi}_{23}$ & $\chi_{82}$ & $2^{5} \cdot 3^{12} \cdot 17$ \\
$\mathrm{Ru}$ & $\chi_{34}$ & $2^{12} \cdot 3^{3}$ & & & \\
\hline
\end{tabular}

is $I$-invariant, we deduce from [7, Theorem 11.7] that $\theta$ extends to $\theta_{0} \in \operatorname{Irr}(I)$. By Gallagher's theorem, $\tau \theta_{0}$ is an irreducible constituent of $\theta^{I}$ for every $\tau \in \operatorname{Irr}(I / M)$, and so $\tau(1) \theta_{0}(1)=\tau(1)$ divides one of the numbers in $\mathcal{A}$. Choose $\tau \in \operatorname{Irr}(I / M)=\operatorname{Irr}\left(\mathrm{Fi}_{23}\right)$ with $\tau(1)=559458900$. This degree divides none of the numbers in $\mathcal{A}$, which is a contradiction. Thus we conclude that $\theta$ is $G^{\prime}$-invariant.

3.2.4. Verifying step 4 . We have shown that $G^{\prime} / M \cong \mathrm{Fi}_{24}^{\prime}$ and for every $\theta \in \operatorname{Irr}(M)$, if $\theta(1)=1$, then $\theta$ is $G^{\prime}$-invariant, so that by Lemma 2.6, $\left|M: M^{\prime}\right|$ divides the order of the Schur multiplier of $\mathrm{Fi}_{24}^{\prime}$. Hence $\left|M: M^{\prime}\right|$ divides 3. Assume first that $M=M^{\prime}$. If $M$ is abelian, then we are done. Assume that $M$ is nonabelian. Let $N \leq M$ be a normal subgroup of $G^{\prime}$ such that $M / N$ is a chief factor of $G^{\prime}$. It follows that $M / N \cong S^{k}$ for some nonabelian simple group $S$. By Lemma 2.4, $S$ possesses a nontrivial irreducible character $\varphi$ such that $\varphi^{k} \in \operatorname{Irr}(M / N)$ extends to $G^{\prime} / N$. Gallagher's theorem yields $\varphi(1)^{k} \tau(1) \in \operatorname{cd}\left(G^{\prime} / N\right) \subseteq \operatorname{cd}\left(G^{\prime}\right)$ for all $\tau \in \operatorname{Irr}\left(G^{\prime} / M\right) \subseteq \operatorname{Irr}\left(G^{\prime} / N\right)$. Since $\operatorname{cd}\left(G^{\prime} / M\right)=\operatorname{cd}(G)$ and $\varphi(1)>1$, if we choose $\tau \in \operatorname{Irr}\left(G^{\prime} / M\right)$ such that $\tau(1)$ is the largest degree of $H$, then $\varphi(1)^{k} \tau(1)$ divides no degree of $G$, a contradiction. If $\left|M: M^{\prime}\right|=3$, then $G^{\prime} / M^{\prime} \cong 3 \cdot \mathrm{Fi}_{24}^{\prime}$. By [3], $3 \cdot \mathrm{Fi}_{24}^{\prime}$ possesses an irreducible character of degree $\chi_{182}(1)=405445459419$ which divides none of the character degrees of $H$. Thus $M=1$.

3.2.5. Verifying step 5. Suppose that $G^{\prime} \times C_{G}\left(G^{\prime}\right) \lessgtr G$ and let $A=C_{G}\left(G^{\prime}\right)$. It follows from step 4 that $G^{\prime} \cong \mathrm{Fi}_{24}^{\prime}$. Then $G / A$ embeds into $\operatorname{Aut}\left(G^{\prime}\right)$ and hence $G$ induces on $G^{\prime}$ some outer automorphism $\sigma$. We have that $\left|\operatorname{Out}\left(\mathrm{Fi}_{24}^{\prime}\right)\right|=2$, and hence $G / A \cong \mathrm{Fi}_{24}^{\prime} \cdot 2$. By [3], $G / A$ possesses an irreducible character of degree 410707651200 which is not a degree of $\mathrm{Fi}_{24}^{\prime}$. Thus $G=G^{\prime} \times A$. Since $A \cong G / G^{\prime}$, we obtain that $A$ is abelian and $G \cong G^{\prime} \times A \cong \mathrm{Fi}_{24}^{\prime} \times A$.

\section{Huppert's conjecture for the sporadic simple group $\mathbf{F i}_{22}$}

4.1. Some properties of $\mathbf{F i}_{\mathbf{2 2}}$. We list relevant properties of the character degree set of the sporadic simple group $H=\mathrm{Fi}_{22}$ by examining [3]. 
Lemma 4.1. Let $H$ be the sporadic simple group $\mathrm{Fi}_{22}$.

(a) The following are isolated degrees of $H$ :

$$
\begin{aligned}
& 2^{17} \cdot 11, \quad 2^{5} \cdot 3^{5} \cdot 5^{2} \cdot 7, \quad 2^{3} \cdot 3^{8} \cdot 11, \\
& 3^{8} \cdot 5 \cdot 11, \quad 3^{9} \cdot 7 \cdot 13, \quad 2^{8} \cdot 5^{2} \cdot 7 \cdot 13 \text {. }
\end{aligned}
$$

(b) If $1 \neq \chi(1) \in \mathrm{cd}(H)$, then $\operatorname{gcd}(7 \cdot 11 \cdot 13, \chi(1))>1$.

(c) The group $H$ has neither proper power degrees nor consecutive degrees.

(d) If $K$ is a maximal subgroup of $H$ such that $|H: K|$ divides some character degree $\chi(1)$ of $H$ then one of the following cases holds.

(a) $K \cong 2 \cdot \mathrm{U}_{6}(2)$ and $\chi(1) /|H: K|$ divides one of the numbers in the set

$$
\mathcal{A}=\left\{2^{2} \cdot 3 \cdot 5 \cdot 11,2^{3} \cdot 5 \cdot 7,3^{5}\right\}
$$

(b) $K \cong \mathrm{O}_{8}^{+}(2): S_{3}$ and $\chi(1) /|H: K|$ divides 6 .

(c) $K \cong 2^{10}: \mathrm{M}_{22}$ and $\chi(1) /|H: K|$ divides 6 .

(e) The Schur multiplier $M\left(\mathrm{Fi}_{22}\right) \cong \mathbb{Z}_{6}$ and outer automorphism group $\operatorname{Out}\left(\mathrm{Fi}_{22}\right) \cong$ $\mathbb{Z}_{2}$.

4.2. Verifying Huppert's conjecture for $\mathbf{F i}_{22}$. Steps 1 and 2 follow in the same way as steps 1 and 2 of Section 3. In this case we use Lemma 4.1 and apply the argument to the isolated degree $3^{8} \cdot 5 \cdot 11$ and use the fact that $\operatorname{gcd}\left(2^{7} \cdot 11,3^{9} \cdot 7 \cdot 13\right)=1$. Hence the chief factor $G^{\prime} / M$ of $G$ is isomorphic to $\mathrm{Fi}_{22}$.

4.2.1. Verifying step 3. Let $\theta \in \operatorname{Irr}(M)$ with $\theta(1)=1$ and let $I=I_{G^{\prime}}(\theta)$. Assume that $I<G^{\prime}$ and $\theta^{I}=\sum_{i=1}^{s} e_{i} \phi_{i}$, where $\phi_{i} \in \operatorname{Irr}(I)$ for $i=1,2, \ldots, s$. Let $U / M$ be a maximal subgroup of $G^{\prime} / M$ containing $I / M$ and let $t=|U: I|$. Then $\phi_{i}(1)\left|G^{\prime}: I\right|$ is a character degree of $G^{\prime}$ by Lemma 2.2(a). Thus $t \phi_{i}(1)\left|G^{\prime}: U\right|$ divides some character degree of $G$ and hence the index $\left|G^{\prime}: U\right|$ must divide some character degree of $H$. By Lemma 4.1(d), one of the following cases holds.

(a) $U / M \cong 2 \cdot \mathrm{U}_{6}(2)$. For each $i, t \phi_{i}(1)$ divides one of the numbers in $\mathcal{A}$. As $U / M$ is perfect, the center of $U / M$ lies in every maximal subgroup of $U / M$ and so the indices of maximal subgroups of $U / M$ and those of $\mathrm{U}_{6}(2)$ are the same. By inspecting the list of maximal subgroups of $\mathrm{U}_{6}(2)$ in [3], the index of a maximal subgroup of $\mathrm{U}_{6}(2)$ divides no number in $\mathcal{A}$. Thus $t=1$ and hence $I=U$. Let $M \leq L \leq I$ such that $L / M$ is isomorphic to the center of $I / M$, and let $\lambda \in \operatorname{Irr}(L \mid \theta)$. As $L \unlhd I$, for any $\varphi \in \operatorname{Irr}(I \mid \lambda)$ we have that $\varphi(1)$ divides one of the numbers in $\mathcal{A}$. As above, we deduce that $\lambda$ is $I$-invariant. Let $L \leq T \leq I$ such that $T / L \cong \mathrm{U}_{5}(2)$. It follows that $\lambda$ is $T$-invariant and since the Schur multiplier of $T / L \cong \mathrm{U}_{5}(2)$ is trivial, $\lambda$ extends to $\lambda_{0} \in \operatorname{Irr}(T)$. By Gallagher's theorem, $\tau \lambda_{0}$ is an irreducible constituent of $\lambda^{T}$ for every $\tau \in \operatorname{Irr}(T / L)$. Choose $\tau \in \operatorname{Irr}(T / L)$ with $\tau(1)=2^{10}$ and let $\gamma=\tau \lambda_{0} \in \operatorname{Irr}(T \mid \lambda)$. If $\chi \in \operatorname{Irr}(I)$ is any irreducible constituent of $\gamma^{I}$, then $\chi(1) \geq \gamma(1)$ by Frobeniusreciprocity [7, Lemma 5.2] 
and $\chi(1)$ divides one of the numbers in $\mathcal{A}$, which implies that $\gamma(1)=2^{10} \lambda(1) \leq \chi(1) \leq$ 660, which is impossible.

(b) $U / M \cong \mathrm{O}_{8}^{+}(2): S_{3}$. For each $i$, $t \phi_{i}$ (1) divides 6 . Let $M \unlhd W \unlhd U$ such that $W / M \cong \mathrm{O}_{8}^{+}(2)$. We have that $M \unlhd I \cap W \unlhd I$ and $M \unlhd I \cap W \leq W$. Assume that $W \not \leq I$. Then $I \leq W I \leq U$ and $t=|U: I|=|U: W I| \cdot|W I: I|$. Now $|W I: I|=|W: W \cap I|>1$, and hence $t$ is divisible by $|W: W \cap I|$. As $W / M \cong \mathrm{O}_{8}^{+}(2), t$ is divisible by the index of some maximal subgroup of $\mathrm{O}_{8}^{+}(2)$. Thus some index of a maximal subgroup of $\mathrm{O}_{8}^{+}(2)$ divides 6 , which is impossible by [3]. Thus $W \leq I \leq U$. Write $\theta^{W}=\sum_{i=1}^{l} f_{i} \mu_{i}$, where $\mu_{i} \in \operatorname{Irr}(W \mid \theta)$. As $W \unlhd I, \mu_{i}(1)$ divides 6 for every $i$. If $f_{j}=1$ for some $j$, then $\theta$ extends to $\theta_{0} \in \operatorname{Irr}(W)$. By Gallagher's theorem, $\tau \theta_{0}$ is an irreducible constituent of $\theta^{W}$ for every $\tau \in \operatorname{Irr}(W / M)$, and so $\tau(1) \theta_{0}(1)=\tau(1)$ divides 6 . However, we can choose $\tau \in \operatorname{Irr}(W / M)$ with $\tau(1)=28$ and this degree does not divide 6 . Therefore $f_{i}>1$, for all $i$. We deduce from Lemma 2.2(c) that, for each $i, f_{i}$ is the degree of a nontrivial proper irreducible projective representation of $\mathrm{O}_{8}^{+}(2)$. As $\mu_{i}(1)=f_{i} \theta(1)=f_{i}$, each $f_{i}$ divides 6 . This is impossible as the smallest nontrivial proper projective degree of $\mathrm{O}_{8}^{+}(2)$ is 8 .

(c) $U / M \cong 2^{10}: \mathrm{M}_{22}$. For each $i, t \phi_{i}(1)$ divides 6 . Let $M \unlhd L \unlhd U$ such that $L / M \cong 2^{10}$. We have that $L \unlhd U$ and $U / L \cong \mathrm{M}_{22}$. The same argument as in part (b) shows that $U=I L$ since the minimal index of a maximal subgroup of $\mathrm{M}_{22}$ is 22 by [3]. Hence $U / L \cong I / L_{1} \cong \mathrm{M}_{22}$, where $L_{1}=L \cap I \unlhd I$. Let $\lambda \in \operatorname{Irr}\left(L_{1} \mid \theta\right)$. Then for any $\varphi \in \operatorname{Irr}(I \mid \lambda)$, we have that $\varphi(1)$ divides 6 . We conclude that $\lambda$ is $I$-invariant as the index of a maximal subgroup of $I / L_{1} \cong \mathrm{M}_{22}$ is at least 22. Write $\lambda^{I}=\sum_{i=1}^{l} f_{i} \mu_{i}$, where $\mu_{i} \in \operatorname{Irr}(I \mid \lambda)$. Then $\mu_{i}(1)$ divides 6 , for each $i$. If $f_{j}=1$ for some $j$, then $\lambda$ extends to $\lambda_{0} \in \operatorname{Irr}(I)$. By Gallagher's theorem $\tau \lambda_{0}$ is an irreducible constituent of $\lambda^{I}$ for every $\tau \in \operatorname{Irr}\left(I / L_{1}\right)$, and so $\tau(1) \lambda_{0}(1)=\tau(1)$ divides 6. However, we can choose $\tau \in \operatorname{Irr}\left(I / L_{1}\right)$ with $\tau(1)=21$ and this degree does not divide 6 . Therefore $f_{i}>1$ for all $i$. We deduce from Lemma 2.2(c) that, for each $i, f_{i}$ is the degree of a nontrivial proper irreducible projective representation of $\mathbf{M}_{22}$. Moreover, as $\mu_{i}(1)=f_{i} \lambda(1)=f_{i}$, each $f_{i}$ divides 6 . However, this is impossible as the smallest nontrivial proper projective degree of $\mathrm{M}_{22}$ is 10 . Therefore $\theta$ is $G^{\prime}$-invariant.

4.2.2. Verifying step 4. Since $G^{\prime} / M \cong \mathrm{Fi}_{22}$ and $I_{G^{\prime}}(\theta)=G^{\prime}$, for every linear character $\theta \in \operatorname{Irr}(M)$, Lemma 2.6 implies that $\left|M: M^{\prime}\right|$ divides 6 , the order of the Schur multiplier of $\mathrm{Fi}_{22}$. Suppose that $M=M^{\prime}$. If $M$ is abelian, then we are done. If $M$ is not abelian, then by the same argument as in step 4 of Section 3, a proper multiple of $\tau(1)=2729376 \in \operatorname{cd}\left(G^{\prime} / M\right)$ divides some degree of $G$, a contradiction. Suppose that $\left|M: M^{\prime}\right|=2,3$ or 6 . We arrive at a contradiction by considering $\chi_{110}(1)=2358720 \in$ $\operatorname{cd}\left(2 \cdot \mathrm{Fi}_{22}\right), \chi_{164}(1)=3088800 \in \operatorname{cd}\left(3 \cdot \mathrm{Fi}_{22}\right)$, and $\chi_{198}(1)=3088800 \in \operatorname{cd}\left(6 \cdot \mathrm{Fi}_{22}\right)$, respectively. Thus $M=1$.

4.2.3. Verifying step 5. This step follows exactly in the same manner as step 5 of Section 3 by considering the degree $1965600 \in \operatorname{cd}\left(\mathrm{Fi}_{22} \cdot 2\right)$. Therefore $G \cong G^{\prime} \times A \cong$ $\mathrm{Fi}_{22} \times A$, where $A$ is abelian. 


\section{Huppert's conjecture for the sporadic simple group $\mathrm{Co}_{1}$}

5.1. Some properties of $\mathrm{Co}_{1}$. We provide relevant properties of the character degree set of the sporadic simple group $H=\mathrm{Co}_{1}$ by examining [3]. Observe that $\pi(H)=$ $\{2,3,5,7,11,13,23\}$.

Lemma 5.1. Let $\mathrm{H}$ be the sporadic simple group $\mathrm{Co}_{1}$.

(a) The following are isolated degrees of $H$ :

$$
\begin{aligned}
& 2^{18} \cdot 3^{6}, \quad 2^{18} \cdot 5^{2} \cdot 7 \cdot 11, \quad 2^{19} \cdot 7 \cdot 11, \\
& 3^{7} \cdot 5^{3} \cdot 7 \cdot 11, \quad 3^{7} \cdot 5^{3} \cdot 7 \cdot 23, \quad 2^{12} \cdot 3 \cdot 5^{3} \cdot 11 \cdot 13 \text {. }
\end{aligned}
$$

(b) If $1 \neq \chi(1) \in \mathrm{cd}(H)$ and $\operatorname{gcd}(11 \cdot 13 \cdot 23, \chi(1))=1$, then $\chi(1)=2^{18} \cdot 3^{6}$.

(c) The group $H$ has neither consecutive degrees nor nontrivial prime power degrees and the only proper nontrivial power degree of $H$ is $2^{18} \cdot 3^{6}$.

(d) If $K$ is a maximal subgroup of $H$ such that $|H: K|$ divides some character degree $\chi(1)$ of $H$, then one of the following cases holds.

(a) $K \cong \mathrm{Co}_{2}$ and $\chi(1) /|H: K|$ divides one of the numbers in the set $\mathcal{A}=$ $\left\{3 \cdot 7 \cdot 11 \cdot 23,2^{3} \cdot 3^{3} \cdot 5\right\}$.

(b) $K \cong 2_{+}^{1+8} \cdot \mathrm{O}_{8}^{+}(2)$ and $\chi(1) /|H: K|$ divides 1 .

(e) The Schur multiplier $M\left(\mathrm{Co}_{1}\right) \cong \mathbb{Z}_{2}$ and outer automorphism group $\operatorname{Out}\left(\mathrm{Co}_{1}\right)$ is trivial.

5.2. Verifying Huppert's conjecture for Con . Steps 1 and 2 follow exactly in the same manner as steps 1 and 2 in Section 3, respectively. We use Lemma 5.1 and apply the argument to the isolated degree $2^{18} \cdot 3^{6}$ and use the fact that $\operatorname{gcd}\left(2^{18} \cdot 3^{6}, 11 \cdot 13\right.$. $23)=1$. Consequently, one can show that the chief factor $G^{\prime} / M$ of $G$ is isomorphic to $\mathrm{Co}_{1}$.

5.2.1. Verifying step 3. Let $\theta \in \operatorname{Irr}(M)$ with $\theta(1)=1$ and let $I=I_{G^{\prime}}(\theta)$. Assume that $I<G^{\prime}$ and $\theta^{I}=\sum_{i=1}^{s} e_{i} \phi_{i}$, where $\phi_{i} \in \operatorname{Irr}(I)$ for $i=1,2, \ldots, s$. Let $U / M$ be a maximal subgroup of $G^{\prime} / M$ containing $I / M$ and let $t=|U: I|$. Then $\phi_{i}(1)\left|G^{\prime}: I\right|$ is a character degree of $G^{\prime}$ by Lemma 2.2(a). Thus $t \phi_{i}(1)\left|G^{\prime}: U\right|$ divides some character degree of $G$ and so the index $\left|G^{\prime}: U\right|$ must divide some character degree of $H$. By Lemma 5.1(d), one of the following cases holds.

(a) $U / M \cong \mathrm{Co}_{2}$. For each $i, t \phi_{i}(1)$ divides one of the numbers in $\mathcal{A}$. By inspecting the list of maximal subgroups of $\mathrm{Co}_{2}$ in [3], the index of a maximal subgroup of $\mathrm{Co}_{2}$ divides no number in $\mathcal{A}$ so that $t=1$ and hence $I=U$. As the Schur multiplier of $I / M \cong \mathrm{Co}_{2}$ is trivial, $\theta$ extends to $\theta_{0} \in \operatorname{Irr}(I)$. By Gallagher's theorem, $\tau \theta_{0}$ is an irreducible constituent of $\theta^{I}$ for every $\tau \in \operatorname{Irr}(I / M)$. Choose $\tau \in \operatorname{Irr}(I / M)$ with $\tau(1)=2^{3} \cdot 11 \cdot 23$ and let $\gamma=\tau \theta_{0} \in \operatorname{Irr}(I \mid \theta)$. Then $\gamma(1)=2^{3} \cdot 11 \cdot 23$ divides no number in $\mathcal{A}$, which is a contradiction.

(b) $U / M \cong 2_{+}^{1+8} \cdot \mathrm{O}_{8}^{+}(2)$. For each $i, t \phi_{i}(1)$ divides 1 . It follows that $t=1$ and all $\phi_{i}(1)=1$. Hence $I=U$ and every irreducible constituent of $\theta^{I}$ is linear so that $\theta$ extends to $\theta_{0} \in \operatorname{Irr}(I)$. By Gallagher's theorem, $\tau \theta_{0}$ are all irreducible 
constituents of $\theta^{I}$, where $\tau \in \operatorname{Irr}(I / M)$. Hence $\tau(1) \theta_{0}(1)=\tau(1)=1$, which implies that all irreducible characters of $I / M$ are linear and so $I / M \cong 2_{+}^{1+8} \cdot \mathrm{O}_{8}^{+}(2)$ is abelian, which is a contradiction. Therefore $\theta$ is $G^{\prime}$-invariant.

5.2.2. Verifying step 4. As $G^{\prime} / M \cong \mathrm{Co}_{1}$ and $I_{G^{\prime}}(\theta)=G^{\prime}$ for every linear character $\theta \in \operatorname{Irr}(M)$, Lemma 2.6 implies that $\left|M: M^{\prime}\right|$ divides 2, the order of the Schur multiplier of $\mathrm{Co}_{1}$. Considering the largest degree $\tau(1)$ of $\mathrm{Co}_{1}$, the same argument as in step 4 of Section 3 shows that if $\left|M: M^{\prime}\right|=1$, then $M$ is abelian and thus $M=1$. If $\left|M: M^{\prime}\right|=2$, then $G^{\prime} / M^{\prime} \cong 2 \cdot \mathrm{Co}_{1}$. By [3], $2 \cdot \mathrm{Co}_{1}$ possesses an irreducible character of degree $\chi_{167}(1)=1021620600$, which divides no degree of $H$. Thus $M=1$.

5.2.3. Verifying step 5. Let $A=C_{G}\left(G^{\prime}\right)$. As Out $\left(\mathrm{Co}_{1}\right)=1$ and $G^{\prime} \cong \mathrm{Co}_{1}$ is simple, we deduce that $G=G^{\prime} A$ and $G^{\prime} \cap A=1$. Therefore $G=G^{\prime} \times A \cong \mathrm{Co}_{1} \times A$, where $A \cong G / G^{\prime}$ is abelian.

\section{Huppert's conjecture for the sporadic simple group $\mathrm{Co}_{2}$}

6.1. Some properties of $\mathrm{Co}_{2}$. We provide relevant properties of the character degree set of the sporadic simple group $H=\mathrm{Co}_{2}$ by examining [3]. Observe that $\pi(H)=$ $\{2,3,5,7,11,23\}$.

\section{Lemma 6.1. Let $\mathrm{H}$ be the sporadic simple group $\mathrm{Co}_{2}$.}

(a) We have that $2^{18} \cdot 7$ is an isolated degree of $H$. Moreover, the two degrees $2^{2} \cdot 3^{5} \cdot 5^{2} \cdot 23$ and $2 \cdot 3^{6} \cdot 5^{3} \cdot 11$ of $H$ are maximal with respect to divisibility and no nontrivial divisor of the greatest common divisor of both of these degrees is a degree of $H$.

(b) If $1 \neq \chi(1) \in \operatorname{cd}(H)$ and $\operatorname{gcd}(11 \cdot 23, \chi(1))=1$, then $\chi(1) \in\left\{3^{6} \cdot 5^{3}, 2^{18} \cdot 7\right\}$.

(c) The group $H$ has no consecutive degrees. The only proper nontrivial power degree of $H$ is $3^{6} \cdot 5^{3}$ and the only prime power degree of $H$ is 23.

(d) If $K$ is a maximal subgroup of $H$ such that $|H: K|$ divides some character degree $\chi(1)$ of $H$, then one of the following cases holds.

(a) $K \cong \mathrm{U}_{6}(2): 2$ and $\chi(1) /|H: K|$ divides one of the numbers in the set $\mathcal{A}_{1}=$ $\left\{2 \cdot 5 \cdot 7 \cdot 11,2^{4} \cdot 5 \cdot 7,3^{5}\right\}$.

(b) $K \cong 2^{10}: \mathrm{M}_{22}: 2$ and $\chi(1) /|H: K|$ divides one of the numbers in the set $\mathcal{A}_{2}=\left\{3^{2} \cdot 5,2^{2} \cdot 3\right\}$.

(c) $K \cong 2_{+}^{1+8}: \mathrm{S}_{6}(2)$ and $\chi(1) /|H: K|$ divides one of the numbers in the set $\mathcal{A}_{3}=\{5 \cdot 7,3 \cdot 5,2\}$.

(d) $K \cong \mathrm{McL}$ and $\chi(1) /|H: K|$ divides $2^{2} \cdot 11$.

(e) The Schur multiplier and the outer automorphism group of $\mathrm{Co}_{2}$ are both trivial.

\subsection{Verifying Huppert's conjecture for $\mathrm{Co}_{2}$.}

6.2.1. Verifying step 1. Suppose that $G^{\prime} \neq G^{\prime \prime}$. Then there exists a normal subgroup $N \unlhd G$ where $N$ is maximal such that $G / N$ is solvable and nonabelian. By Lemma 2.3, $G / N$ is either an $r$-group for some prime $r$ or a Frobenius group. 
Case 1. $G / N$ is an $r$-group. Then there exists $\psi \in \operatorname{Irr}(G / N)$ such that $\psi(1)=r^{b}>1$. By Lemma 6.1(c), we obtain that $r^{b}=23$. Let $\chi \in \operatorname{Irr}(G)$ such that $\chi(1)=2^{18} \cdot 7$. As $\operatorname{gcd}(|G / N|, \chi(1))=1$, by Lemma 2.1, we have that $\chi_{N} \in \operatorname{Irr}(N)$. By Gallagher's theorem, $\chi \psi \in \operatorname{Irr}(G)$ so that $\chi(1) \psi(1) \in \operatorname{cd}(G)$, which is impossible as $\chi(1)$ is an isolated degree of $G$ by Lemma 6.1(a).

Case 2. $G / N$ is a Frobenius group with Frobenius kernel $F / N,|F / N|=r^{a}, 1<f=\mid G$ : $F \mid \in \operatorname{cd}(G)$ and $r^{a} \equiv 1 \bmod f$. By Lemma 2.3(b), if $\chi \in \operatorname{Irr}(G)$ such that $\chi(1)$ is isolated, then either $f=\chi(1)$ or $r \mid \chi(1)$. By Lemma 6.1(a), $2^{18} \cdot 7$ is an isolated degree of $G$ so that either $f=2^{18} \cdot 7$ or $r \in\{2,7\}$. Assume first that $f=2^{18} \cdot 7$. By Lemma 2.3(b) again, if $\chi \in \operatorname{Irr}(G)$ with $r \nmid \chi(1)$, then $\chi(1) \mid f$. As $f$ is isolated we deduce that $r$ must divide every nontrivial degree $\chi(1)$ of $G$ such that $\chi(1) \neq f$. However, $G$ possesses two relatively prime degrees 23 and $5^{2} \cdot 11$ different from $f$, which leads to a contradiction. Hence $r \in\{2,7\}$. By Lemma 6.1(a), the degrees $\chi_{1}(1)=2^{2} \cdot 3^{5} \cdot 5^{2} \cdot 23$ and $\chi_{2}(1)=2 \cdot 3^{6} \cdot 5^{3} \cdot 11$ of $G$ are maximal with respect to divisibility and are both prime to 7, so that if $r=7$, then by Lemma 2.3(b), $f$ must divide both of these degrees. This is impossible by Lemma 6.1(a) and thus $r=2$. By Lemma 2.3(b) again, if $r^{a}$ divides $\chi_{i}(1)^{2}$ for $i=1$ or 2 , then $r^{a} \leq 2^{4}$ and hence $f \leq r^{a}-1 \leq 2^{4}-1=15$, which is impossible as the smallest nontrivial degree of $\mathrm{Co}_{2}$ is 23 . Thus we conclude that $f$ divides both $\chi_{i}(1)$, which again contradicts Lemma 6.1(a). Thus $G^{\prime}=G^{\prime \prime}$.

6.2.2. Verifying step 2. Let $M \leq G^{\prime}$ be a normal subgroup of $G$ such that $G^{\prime} / M$ is a chief factor of $G$. As $G^{\prime}$ is perfect, $G^{\prime} / M$ is nonabelian. Hence $G^{\prime} / M \cong S^{k}$ for some nonabelian simple group $S$ and some integer $k \geq 1$.

Claim 1. $S$ is not an alternating group of degree at least seven. If $S=\mathrm{A}_{n}$ for $n \geq 7$, then by Lemma 2.4, two nontrivial irreducible characters $\theta_{1}, \theta_{2}$ of consecutive degrees $\theta_{1}(1)=n(n-3) / 2, \theta_{2}(1)=\theta_{1}(1)+1$ extend to $\operatorname{Aut}(S)$, so that by Lemma 2.5, $\theta_{i}^{k}(1) \in \operatorname{cd}(G)$. By Lemma 6.1(c), $G$ has only one nontrivial power degree, so that $k=1$. But then $G$ possesses two consecutive nontrivial character degrees, contradicting Lemma 6.1(c).

Claim 2. $S$ is not a simple group of Lie type. If $S$ is a simple group of Lie type in characteristic $p$ and $S \neq{ }^{2} \mathrm{~F}_{4}(2)^{\prime}$, then the Steinberg character of $S$ of degree $|S|_{p}$ extends to $\operatorname{Aut}(S)$. By Lemma 2.5, $|S|_{p}^{k} \in \operatorname{cd}(G)$ and hence $G$ possesses a nontrivial prime power degree. By Lemma 6.1(c), we deduce that $|S|_{p}^{k}=23$, and so $k=1, p=23$ and $|S|_{p}=23$. It follows that the Lie rank of $S$ is 1 and the only possibility for $S$ is $\mathrm{L}_{2}(23)$. By [3], $\mathrm{L}_{2}(23)$ possesses an irreducible character of degree 22 which is extendible to $\operatorname{Aut}(S)$. By Lemma 2.5, $G$ has an irreducible character of degree 22, which is a contradiction.

Claim 3. $S \cong \mathrm{Co}_{2}$. By Claims $1-3, S$ is a sporadic simple group or the Tits group. If $k \geq 2$, Lemmas 2.4(b) and 2.5 imply that $G$ would possess two distinct nontrivial proper power degrees, which contradicts Lemma 6.1(c). Thus $k=1$. As $\pi(S) \subseteq \pi(H)=$ $\{2,3,5,7,11,13,23\}$, we only need to consider the simple groups in Table 1 excluding $\mathrm{Co}_{2}$. For each such simple group $S$, there exists an irreducible character $\theta \in \operatorname{Irr}(S)$ 
which is extendible to $\operatorname{Aut}(S)$. By Lemma 2.5, we obtain that $\theta(1) \in \operatorname{cd}(G)$ with $\operatorname{gcd}(\theta(1), 11 \cdot 13 \cdot 23)=1$. It follows from Lemma $6.1\left(\right.$ b) that $\theta(1) \in\left\{3^{6} \cdot 5^{3}, 2^{18} \cdot 7\right\}$, which is impossible by inspecting Table 1 .

6.2.3. Verifying step 3. Let $\theta \in \operatorname{Irr}(M)$ with $\theta(1)=1$ and let $I=I_{G^{\prime}}(\theta)$. Assume that $I<G^{\prime}$ and $\theta^{I}=\sum_{i=1}^{s} e_{i} \phi_{i}$, where $\phi_{i} \in \operatorname{Irr}(I)$ for $i=1,2, \ldots, s$. Let $U / M$ be a maximal subgroup of $G^{\prime} / M$ containing $I / M$ and let $t=|U: I|$. Then $\phi_{i}(1)\left|G^{\prime}: I\right|$ is a character degree of $G^{\prime}$ by Lemma 2.2(a), so it divides some character degree of $G$. Thus $t \phi_{i}(1)\left|G^{\prime}: U\right|$ divides some character degree of $G$ and the index $\left|G^{\prime}: U\right|$ must divide some character degree of $H$. By Lemma 6.1(d), one of the following cases holds.

(a) $U / M \cong \mathrm{U}_{6}(2): 2$. For each $i, t \phi_{i}(1)$ divides one of the numbers in $\mathcal{A}_{1}$. Let $M \leq W \leq U$ such that $W / M \cong \mathrm{U}_{6}(2)$. Then $W \unlhd U$. Assume that $W \not I I$. Since $t=|U: I|=|U: W I| \cdot|W I: I|$ and $|W I: I|=|W: W \cap I|$, the index of some maximal subgroup of $W / M \cong \mathrm{U}_{6}(2)$ divides $t$ and so divides some number in $\mathcal{A}_{1}$, which is a contradiction by [3]. Thus $W \leq I \leq U$. Let $M \leq V \leq W$ such that $V / M \cong \mathrm{U}_{5}(2)$. We have that $\theta$ is $V$-invariant and, since the Schur multiplier of $V / M$ is trivial, $\theta$ extends to $\theta_{0} \in \operatorname{Irr}(V)$. By Gallagher's theorem, $\tau \theta_{0}$ is an irreducible constituent of $\theta^{V}$ for every $\tau \in \operatorname{Irr}(V / M)$. Choose $\tau \in \operatorname{Irr}(V / M)$ with $\tau(1)=1215$ and let $\gamma=\tau \theta_{0} \in \operatorname{Irr}(V \mid \theta)$. If $\chi \in$ $\operatorname{Irr}(I)$ is an irreducible constituent of $\gamma^{I}$, then $\chi(1) \geq \gamma(1)$ by Frobenius reciprocity [7, Lemma 5.2] and also $\chi(1)$ divides one of the numbers in $\mathcal{A}_{1}$, which implies that $\gamma(1)=1215 \leq \chi(1) \leq 770$, which is impossible.

(b) $U / M \cong 2^{10}: \mathrm{M}_{22}: 2$. For each $i$, $t \phi_{i}(1)$ divides one of the numbers in $\mathcal{A}_{2}$. Let $M \unlhd L \unlhd W \unlhd U$ be a subgroup of $U$ such that $L / M \cong 2^{10}$ and $W / L \cong M_{22}$. Let $L_{1}=L \cap I$ and $X=W \cap I$. As $I \leq W I \leq U$, we obtain $t=|U: I|=|U: W I| \cdot|W I: I|=$ $|U: W I| \cdot|W: X|$. Now $X \leq X L \leq W$ so that $|W: X|=|W: X L| \cdot|X L: X|$. Assume that $|W: X L|>1$. Then $L \leq X L \leq W$ and $W / L \cong \mathrm{M}_{22}$ so an index of a maximal subgroup of $W / L \cong \mathrm{M}_{22}$ must divide a number in $\mathcal{A}_{2}$. This is impossible by checking [3]. Thus $W=X L$ and $W / L \cong X / L_{1} \cong \mathrm{M}_{22}$. Let $\lambda \in \operatorname{Irr}\left(L_{1} \mid \theta\right)$. As $X=W \cap I \unlhd I$, we deduce that for any irreducible constituent $\varphi$ of $\lambda^{X}, \varphi(1)$ divides 12 or 45 . Write $\lambda^{X}=\sum_{i=1}^{l} f_{i} \mu_{i}$, where $\mu_{i} \in \operatorname{Irr}(X \mid \lambda)$. If $f_{j}=1$ for some $j$ then $\lambda$ extends to $\lambda_{0} \in \operatorname{Irr}(X)$. By Gallagher's theorem, $\tau \lambda_{0}$ is an irreducible constituent of $\lambda^{X}$ for every $\tau \in \operatorname{Irr}\left(X / L_{1}\right)$ and $\tau(1) \lambda_{0}(1)=\tau(1)$ divides 45 or 12 . However, we can choose $\tau \in \operatorname{Irr}\left(X / L_{1}\right)$ with $\tau(1)=385$ and this degree does not divide 45 and 12. Therefore $f_{i}>1$ for all $i$. We deduce from Lemma 2.2(c) that, for each $i, f_{i}$ is the degree of a nontrivial proper irreducible projective representation of $\mathbf{M}_{22}$. As $\mu_{i}(1)=f_{i} \theta(1)=f_{i}$, each $f_{i}$ divides 45 or 12. By [3], we obtain that all $f_{i}=45$. As $\left|X / L_{1}\right|=\sum_{i=1}^{l} f_{i}^{2}$, we conclude that $2^{7} \cdot 3^{2} \cdot 5 \cdot 7 \cdot 11=3^{4} \cdot 5^{2} l$, which is impossible.

(c) $U / M \cong 2_{+}^{1+8}: \mathrm{S}_{6}(2)$ For each $i, t \phi_{i}(1)$ divides one of the numbers in $\mathcal{A}_{3}$. Let $M \unlhd L \unlhd U$ be subgroup of $U$ such that $L / M \cong 2_{+}^{1+8}$. Let $L_{1}=L \cap I$ and $X=U \cap I$. As $I \leq I L \leq U$, we obtain $t=|U: I|=|U: I L| \cdot|I L: I|$. Now $L \leq I L \leq U$ and $U / L \cong \mathrm{S}_{6}(2)$. Assume that $|U: I L|>1$. The index of some maximal subgroup of $U / L \cong \mathrm{S}_{6}(2)$ must divide some number in $\mathcal{A}_{3}$. This is impossible by checking [3]. Thus $U=I L$ and 
then $U / L \cong I / L_{1} \cong \mathrm{S}_{6}(2)$. Let $\lambda \in \operatorname{Irr}\left(L_{1} \mid \theta\right)$. Write $\lambda^{I}=\sum_{i=1}^{l} f_{i} \mu_{i}$, where $\mu_{i} \in \operatorname{Irr}(I \mid \lambda)$. If $f_{j}=1$ for some $j$ then $\lambda$ extends to $\lambda_{0} \in \operatorname{Irr}(I)$. By Gallagher's theorem, $\tau \lambda_{0}$ is an irreducible constituent of $\lambda^{I}$ for every $\tau \in \operatorname{Irr}\left(I / L_{1}\right)$ and then $\tau(1) \lambda_{0}(1)=\tau(1)$ divides some number in $\mathcal{A}_{3}$. However, we can choose $\tau \in \operatorname{Irr}\left(I / L_{1}\right)$ with $\tau(1)=512$ and this degree divides no number in $\mathcal{A}_{3}$. Therefore $f_{i}>1$ for all $i$. It follows from Lemma 2.2(c) that $f_{i}$ is the degree of a nontrivial proper irreducible projective representation of $\mathrm{S}_{6}(2)$ for each $i$. As $\mu_{i}(1)=f_{i} \theta(1)=f_{i}$, each $f_{i}$ divides 2,15 or 35 . This is impossible by checking [3].

(d) $U / M \cong \mathrm{McL}$. For each $i, t \phi_{i}(1)$ divides 44 . By inspecting the list of maximal subgroups of McL in [3], no index of a maximal subgroup of McL divides 44 so that $t=1$ and hence $I=U$. Recall that $\theta^{I}=\sum_{i=1}^{s} e_{i} \phi_{i}$, where $\phi_{i} \in \operatorname{Irr}(I)$ for $i=1,2, \ldots, s$. Assume first that $e_{j}=1$ for some $j$. Then $\theta$ extends to $\theta_{0} \in \operatorname{Irr}(I)$. By Gallagher's theorem, $\tau \theta_{0}$ is an irreducible constituent of $\theta^{I}$ for every $\tau \in \operatorname{Irr}(I / M)$ and then $\tau(1) \theta_{0}(1)=\tau(1)$ divides 44. We can choose $\tau \in \operatorname{Irr}(I / M)=\operatorname{Irr}(\mathrm{McL})$ with $\tau(1)=231$ and this degree does not divide 44. Therefore $e_{i}>1$ for all $i$. We deduce from Lemma 2.2(c) that, for each $i, e_{i}$ is the degree of a nontrivial proper irreducible projective representation of McL. As $\phi_{i}(1)=e_{i} \theta(1)=e_{i}$, each $e_{i}$ divides 44. It follows that $e_{i} \leq 44$ for each $i$ and $e_{i}$ is the degree of a nontrivial proper irreducible projective representation of McL. Using [3], we deduce that there is no such projective degree. Therefore $\theta$ is $G^{\prime}$-invariant.

6.2.4. Verifying steps 4 and 5. Since $G^{\prime} / M \cong \mathrm{Co}_{2}$ and $I_{G^{\prime}}(\theta)=G^{\prime}$ for linear characters $\theta \in \operatorname{Irr}(M)$, by Lemma 2.6, $\left|M: M^{\prime}\right|$ divides the order of the Schur multiplier of $\mathrm{Co}_{2}$, which is 1 . Thus $M=M^{\prime}$. By considering the largest degree $\tau(1)$ of $\mathrm{Co}_{2}$, the same argument as in step 4 of Section 3 shows that $M$ is abelian. Therefore $M=1$. Since $\operatorname{Out}\left(\mathrm{Co}_{2}\right)=1$, step 5 follows in the same way as step 5 of Section 5 .

\section{Huppert's conjecture for the sporadic simple group $\mathrm{Co}_{3}$}

7.1. Some properties of $\mathbf{C o}_{3}$. We provide relevant properties of the character degree set of the sporadic simple group $H=\mathrm{Co}_{3}$ by examining [3]. Observe that $\pi(H)=$ $\{2,3,5,7,11,23\}$.

Lemma 7.1. Let $\mathrm{H}$ be the sporadic simple group $\mathrm{Co}_{3}$.

(a) We have that $2^{7} \cdot 3^{6}$ and $3^{6} \cdot 5^{3}$ are isolated degrees of $H$. The degrees $2^{7} \cdot 5^{2} \cdot 23$, $2^{7} \cdot 5^{2} \cdot 7 \cdot 11$, and $5^{3} \cdot 7 \cdot 11 \cdot 23$ of $H$ are maximal with respect to divisibility. No nontrivial divisor of both $2^{7} \cdot 5^{2} \cdot 23$ and $2^{7} \cdot 5^{2} \cdot 7 \cdot 11$ is a degree of $H$.

(b) If $1 \neq \chi(1) \in \operatorname{cd}(H)$ and $\operatorname{gcd}(11 \cdot 23, \chi(1))=1$ then $\chi(1) \in\left\{2^{7} \cdot 3^{6}, 3^{6} \cdot 5^{3}, 2^{7} \cdot 7\right\}$.

(c) The group $H$ has no consecutive degrees. The only proper nontrivial power degree of $H$ is $3^{6} \cdot 5^{3}$ and the only prime power degree of $H$ is 23.

(d) If $K$ is a maximal subgroup of $H$ such that $|H: K|$ divides some character degree $\chi(1)$ of $H$ then $K \cong \mathrm{McL}: 2$ and $\chi(1) /|H: K|$ divides one of the numbers in the set $\mathcal{A}=\left\{2^{2} \cdot 3 \cdot 7 \cdot 11,2 \cdot 3 \cdot 5 \cdot 7\right\}$.

(e) The Schur multiplier and the outer automorphism group of $\mathrm{Co}_{3}$ are both trivial. 


\subsection{Verifying Huppert's conjecture for $\mathrm{Co}_{3}$.}

7.2.1. Verifying step 1. This step follows similarly to step 1 of Section 6. We use Lemmas 2.3 and 7.1. If $G / N$ is an $r$-group, we make the same argument by taking $\chi(1)=2^{7} \cdot 3^{6}$. If $G / N$ is a Frobenius group with Frobenius kernel $F / N,|F / N|=r^{a}$ and $1<f=|G: F| \in \operatorname{cd}(G)$, then applying Lemma 2.3(b) to $\chi$, we deduce that either $f=2^{7} \cdot 3^{6}$ or $r \in\{2,3\}$. If $f=2^{7} \cdot 3^{6}$, then again by Lemma 2.3(b), two relatively prime degrees 23 and $5^{2} \cdot 11$ lead us to a contradiction. If $r \in\{2,3\}$, by considering $\chi_{1}(1)=2^{7} \cdot 5^{2} \cdot 23$ and $\chi_{2}(1)=2^{7} \cdot 5^{2} \cdot 7 \cdot 11$, we deduce that $r=2$. In this case, the degrees $3^{6} \cdot 5^{3}$ and $5^{3} \cdot 7 \cdot 11 \cdot 23$ lead us to a contradiction. Thus $G^{\prime}=G^{\prime \prime}$.

7.2.2. Verifying step 2. The same argument as in step 2 of Section 6 shows that the chief factor $G^{\prime} / M$ of $G$ is isomorphic to $\mathrm{Co}_{3}$. To eliminate sporadic simple groups except for $\mathrm{Co}_{3}$, use Lemma 7.1(b) and the fact that $\mathrm{Co}_{2}$ possesses an irreducible character of degree $\chi_{60}(1)=2095875$ which divides no degree of $\mathrm{Co}_{3}$.

7.2.3. Verifying step 3. Let $\theta \in \operatorname{Irr}(M)$ with $\theta(1)=1$ and let $I=I_{G^{\prime}}(\theta)$. Assume that $I<G^{\prime}$ and $\theta^{I}=\sum_{i=1}^{s} e_{i} \phi_{i}$, where $\phi_{i} \in \operatorname{Irr}(I)$ for $i=1,2, \ldots, s$. Let $U / M$ be a maximal subgroup of $G^{\prime} / M$ containing $I / M$ and let $t=|U: I|$. Then $\phi_{i}(1)\left|G^{\prime}: I\right|$ is a character degree of $G^{\prime}$ by Lemma 2.2(a), so it divides some character degree of $G$. Thus $t \phi_{i}(1)\left|G^{\prime}: U\right|$ divides some character degree of $G$ and the index $\left|G^{\prime}: U\right|$ must divide some character degree of $H$. By Lemma 7.1(d), $U / M \cong \mathrm{McL}: 2$. Then for each $i, t \phi_{i}(1)$ divides one of the numbers in $\mathcal{A}$. Let $M \leq W \leq U$ such that $W / M \cong \mathrm{McL}$. Suppose that $W \not \leq I$. Since $I \leq W I \leq U$ and $|W I: I|=|W: W \cap I|$, we deduce that the index of some maximal subgroup of $W / M \cong \mathrm{McL}$ divides $t$, and so divides some number in $\mathcal{A}$, which is a contradiction by [3]. Thus $W \leq I \leq U$. Write $\theta^{W}=\sum_{i=1}^{l} f_{i} \mu_{i}$, where $\mu_{i} \in \operatorname{Irr}(W)$ for $i=1,2, \ldots, s$. As $W \unlhd I$, we deduce that, for each $i, \mu_{i}(1)$ divides one of the numbers in $\mathcal{A}$. Assume first that $f_{j}=1$ for some $j$. Then $\theta$ extends to $\theta_{0} \in \operatorname{Irr}(W)$. By Gallagher's theorem, $\tau \theta_{0}$ is an irreducible constituent of $\theta^{W}$ for every $\tau \in \operatorname{Irr}(W / M)$ and $\tau(1) \theta_{0}(1)=\tau(1)$ divides one of the numbers in $\mathcal{A}$. However, we can choose $\tau \in \operatorname{Irr}(I / M)=\operatorname{Irr}(\mathrm{McL})$ with $\tau(1)=10395$ and this degree divides none of the numbers in $\mathcal{A}$. Therefore $f_{i}>1$ for all $i$. We deduce that, for each $i, f_{i}$ is the degree of a nontrivial proper irreducible projective representation of McL. As $\mu_{i}(1)=f_{i} \theta(1)=f_{i}$, each $f_{i}$ divides one of the numbers in $\mathcal{A}$. It follows that, for each $i, f_{i} \leq 924, f_{i}$ is the degree of a nontrivial proper irreducible projective representation of McL, and $f_{i}$ divides 210 or 924 . Using [3], no such projective degree exists. Thus $\theta$ is $G^{\prime}$-invariant.

7.2.4. Verifying steps 4 and 5. Note that the order of the Schur multiplier of $\mathrm{Co}_{3}$ is 1. Applying Lemma 2.6 and considering the largest degree $\tau(1)$ of $\mathrm{Co}_{3}$, the same argument as in step 4 of Section 3 shows that $M=1$. Since $\operatorname{Out}\left(\mathrm{Co}_{3}\right)=1$, step 5 follows exactly in the same manner as step 5 in Section 5.

\section{Acknowledgements}

The authors are grateful to the anonymous referees and the editor for careful reading of the manuscript and for numerous corrections and suggestions. 


\section{References}

[1] S. H. Alavi, A. Daneshkhah, H. P. Tong-Viet and T. P. Wakefield, 'Huppert's conjecture for $\mathrm{Fi}_{23}$ ', Rend. Semin. Mat. Univ. Padova 126 (2011), 201-211.

[2] M. Bianchi, D. Chillag, M. L. Lewis and E. Pacifici, 'Character degree graphs that are complete graphs', Proc. Amer. Math. Soc. 135(3) (2007), 671-676.

[3] J. H. Conway, R. T. Curtis, S. P. Norton, R. A. Parker and R. A. Wilson, Atlas of Finite Groups (Clarendon Press, Oxford, 1985).

[4] The GAP Group, 'GAP - groups, algorithms, and programming, version 4.4.12', 2008, http://www.gap-system.org.

[5] B. Huppert, Character Theory of Finite Groups, de Gruyter Expositions in Mathematics, 25 (Walter de Gruyter \& Co., Berlin, 1998).

[6] B. Huppert, 'Some simple groups which are determined by the set of their character degrees I', Illinois J. Math. 44 (2000), 828-842.

[7] I. M. Isaacs, Character Theory of Finite Groups (AMS Chelsea Publishing, Providence, RI, 2006).

[8] H. P. Tong-Viet and T. P. Wakefield, 'On Huppert's conjecture for the Monster and Baby Monster', Monatsh. Math. 167(3-4) (2012), 589-600.

S. H. ALAVI, Department of Mathematics, Faculty of Science, Bu-Ali Sina University, Hamedan, Iran

e-mail: alavi.s.hassan@gmail.com, alavi.s.hassan@basu.ac.ir

A. DANESHKHAH, Department of Mathematics, Faculty of Science, Bu-Ali Sina University, Hamedan, Iran

e-mail: daneshkhah.ashraf@gmail.com, adanesh@basu.ac.ir

H. P. TONG-VIET, School of Mathematics, Statistics and Computer Science, University of KwaZulu-Natal, Pietermaritzburg 3209, South Africa

e-mail: Tongviet@ukzn.ac.za

T. P. WAKEFIELD, Department of Mathematics and Statistics, Youngstown State University, Youngstown, Ohio 44555, USA

e-mail: tpwakefield@ysu.edu 